\title{
Homocysteine in pregnancies complicated by preeclampsia with and without IUGR: a comparison with normotensive pregnant women with isolated IUGR and healthy pregnant women
}

\author{
Marzena Laskowska*, Jan Oleszczuk \\ Chair and Department of Obstetrics and Perinatology, Medical University of Lublin, Lublin, Poland. \\ Email: ${ }^{*}$ melaskowska@go2.pl
}

Received 8 October 2011; revised 18 November 2011; accepted 29 November 2011.

\begin{abstract}
Objective: The aim of this study was to analyze homocysteine levels in maternal serum in women with pregnancies complicated by preeclampsia and/or IUGR. Patients and methods: The study was carried out on 49 pregnant patients with normotensive pregnancies complicated by intrauterine fetal growth restriction (group IUGR), 31 patients with preeclampsia complicated by IUGR (group PRE-IUGR), and 35 preeclamptic patients with appropriate-for-gestational-age weight fetuses (group PRE). The control group consisted of $\mathbf{4 7}$ healthy normotensive pregnant patients with singleton uncomplicated pregnancies and with proper intrauterine fetal growth. Results: We revealed higher levels of maternal serum homocysteine in the group of pregnant patients with isolated fetal intrauterine growth restriction in comparison with the control subjects. The concentrations of homocysteine were also higher in both groups of patients with pregnancy complicated by preeclampsia with and without IUGR. The highest levels of homocysteine were observed in preeclamptic women with appropriatefor-gestational-age fetal growth. The mean values were $9.004+/-2.820 \mu \mathrm{mol} / \mathrm{L}$ in the IUGR group, 10.815 $+/-3.785 \mu \mathrm{mol} / \mathrm{L}$ in the group PRE, $9.808+/-2.543$ $\mu \mathrm{mol} / \mathrm{L}$ in the group PRE-IUGR and $7.639+/-2.728$ $\mu \mathrm{mol} / \mathrm{L}$ in the control group. Conclusions: Increased levels of homocysteine are involved in pathogenesis IUGR and preeclampsia and may contribute to endothelial cells activation or dysfunction observed in these pregnancy disorders. Further studies are needed to explain these aspects in order to improve the management and therapeutic strategies for pregnancies complicated by IUGR and/or preeclampsia.
\end{abstract}

Keywords: Homocysteine; Intrauterine Fetal Growth
Restriction (IUGR); Preeclampsia

\section{INTRODUCTION}

Intrauterine fetal growth restriction (IUGR) complicates $8 \%$ of all pregnancies and is a major cause of perinatal mortality and morbidity [1]. One of the most common maternal conditions associated with fetal growth restricttion is maternal hypertension and especially preeclampsia, which complicates $5 \%-10 \%$ of all pregnancies [2]. But it is important that in many cases of pregnancies complicated by IUGR there are an idiopathic placental failure with abnormal placental development and suboptimal fetal nutrition and oxygenation without maternal hypertension or preeclampsia $[3,4]$.

Pathophysiological processes underlying intrauterine fetal growth restriction and/or preeclampsia are complicated, multifactorial and unclear [5]. The impaired invasion of uteroplacental arteries causes changes in the placental and fetal circulatory system and may be the cause of the pathological structure and function of the placenta, resulting in the inadequate fetal oxygenation and nutriation, and consequently leading to preeclampsia or intrauterine fetal growth restriction. [5-7].

Endothelial dysfunction has been hypotheses to play a central role in the pathogenesis of preeclampsia [8-10]. Animal studies suggest that hyperhomocysteinemia affects the blood vessel wall and causes a change in the endothelium and smooth muscle proliferation [11-13].

Hypehomocysteinemia is a risk factor of cardiovascular diseases and vasculopathy. It may be a cause of changes and lesions in endothelial cells due to vascular fibrosis and resulting in the activation of thrombogenesis. Hyperhomocysteinemia promotes oxidation of LDL cholesterol molecules and alterations in the coagulation system and enhances platelet activation [11].

The aim of this study was to analyze homocysteine le- 
vels in maternal serum in pregnancies complicated by intrauterine fetal growth restriction with and without preeclampsia, and in preeclamptic pregnancies with the appropriate fetal growth, and in the control group of healthy pregnancies with normal fetal growth. The study was accepted by the local Ethics Committee (KE-0254/51/ 2010).

\section{PATIENTS AND METHODS}

The study was carried out on 49 pregnant women with normotensive pregnancies complicated by intrauterine growth restricted fetuses (IUGR group), and 66 patients with pregnancy complicated by severe preeclampsia. In the group of preeclamptic women were 31 patients with preeclampsia complicated by intrauterine fetal growth restriction (PRE-IUGR group) and 35 preeclamptic patients with appropriate-for-gestational-age fetuses (PRE group).

Among preeclamptic women there were 6 patients with HELLP syndrome (all in the PRE-IUGR group) and 4 patients with eclampsia ( 3 in the PRE-IUGR group and 1 in the PRE group). One patient from the IUGR group had fetal intrauterine death in the week $38^{\text {th }}$ of gestation. The weight of her stillborn infant was $2060 \mathrm{~g}$.

The control group consisted of 47 healthy normotensive pregnant patients with singleton uncomplicated pregnancies without any renal, cardiac and vascular diseases and with normal laboratory tests and with appropriate-for-gestational-age weight infants (CON group).

Fetal biometry was based on non-invasive ultrasound method and comprised the estimation of gestational age in early gestation and diagnosis of fetal intrauterine growth restriction by monitoring fetal growth later in the second or third trimester of pregnancy.

Intrauterine growth restricted fetuses (IUGR) were classified as such according to ultrasonographic measurement when the weight of the fetus was lower than expected in relation to the gestational age, as determined by the standard curves characteristic of the Polish population, when the fetus was below the $5^{\text {th }}$ centile for gestational age and diagnosis was confirmed by the infant's weight at birth according to the Bretelle et al. [5].

The exclusion criteria for women whose pregnancies were complicated by IUGR fetuses were the presence of a congenital malformation or chromosomal abnormality in the fetus, recent cytomegalovirus infection or drug or alcohol abuse during pregnancy. Pregnant women with multiple pregnancies were also excluded from this study.

Preeclampsia was diagnosed by the increased blood pressure of $>140 \mathrm{mmHg}$ systolic and $>90 \mathrm{mmHg}$ diastolic in women who were normotensive before 20 weeks of gestation accompanied by proteinuria, defined as the urinary excretion of $>0.3 \mathrm{~g}$ protein in $24 \mathrm{~h}$ specimen.
Severe preeclampsia was defined as blood pressure $>160 / 110 \mathrm{mmHg}$ on at least 2 occasions $6 \mathrm{~h}$ apart with proteinuria $>2 \mathrm{~g}$ in a 24 hour urinary protein excretion, and when hypertension and proteinuria were associated with one or more of the following clinical manifestations: renal abnormalities, hematological abnormalities (thrombocytopenia and microangiopathic hemolysis) or HELLP syndrome (hemolysis, elevated liver enzymes, low platelet count and right-upper quadrant pain), or neurologycal symptoms (headache, visual disturbances and seizures). None of the pregnant patients with preeclampsia were affected by chronic hypertension, renal disorders and/or proteinuria before pregnancy and all were normotensive before $20^{\text {th }}$ week of pregnancy.

Preeclamptic patients were admitted to the Department of Obstetrics and Perinatology in the Medical University Hospital in Lublin because of the symptoms of the disease and without signs of labour. All preeclamptic women were normotensive 3 months after delivery.

All arterial blood pressure measurements in the control group and in the group of patients with isolated fetal intrauterine growth restriction were normal and did not exceed 135/85 $\mathrm{mmHg}$. None of the patients from any of these groups suffered from proteinuria.

All patients in the study were non-smokers. An informed consent from all studied patients was obtained for peripheral blood sampling. Five milliliters of blood were collected by venipuncture from each preeclamptic patient and from each woman from the control group and placed in sterile tubes. They were centrifuged for $15 \mathrm{~min}$ at $500 \mathrm{xg}$. The homocysteine concentrations were estimated using an ELISA assay according to the manufacturer's instructions (human sandwich ELISA kit AxisShield Diagnostics Ltd, UK)).

In the statistical analysis results were expressed as mean +/- SD or SEM or as median values and were statistically analyzed with the computer program "Statistica" using the Shapiro-Wilk test for the normal distribution of data, and equality of variance by Levene test and, subsequently two-tailed Student's t-tests, or (in unequal variance) the Cochran-Cox test.

The Analysis of Variance-ANOVA and Kruskal-Wallis tests were used to test for differences among four independent groups. A statistically significant effect in ANOVA was followed up with follow-up post hoc Tukey's test in order to assess which group is different from which other groups. A $p$-value of less than 0.05 was considered to be significant.

\section{RESULTS}

There were no statistically significant differences in gravidity, parity, maternal age and height in patient's profiles between groups. Maternal weight and BMI were 
lower in the group of patients with pregnancy complicated by isolated IUGR than in the control group and also in comparison with both groups of preeclamptic women.

Systolic and diastolic blood pressure were higher in both study groups of preeclamptic pregnant women than in the control group and than in pregnancies complicated by isolated fetal growth restriction. These differences were statistically significant ( $\mathrm{p}<0.000001)$.

There were no statistically significant differences in age of gestation between both groups of preeclamptic patients, but significantly lower birth weight of infants was observed in preeclamptic patients with IUGR. The age of gestation was lower in both preeclamptic groups of pregnant patients also in comparison with normotensive women with pregnancy complicated by intrauterine fetal growth restriction. But birth weight of infants was lowest in the group PRE-IUGR and was significantly lower in comparison with group PRE, group IUGR and than in the control subjects. Birth weight of infants in the group of patients with pregnancy complicated by isolated IUGR was also lower than in group PRE, but this difference was not statistically significant in spite of significantly higher age of pregnancy in group IUGR.

Results are presented in Table 1.
The pregnant normotensive patients with isolated fetal growth restriction revealed higher levels of maternal serum homocysteine in comparison with the control subjects.

The concentrations of homocysteine in maternal serum were also higher in both groups of women with pregnancies complicated by preeclampsia. The highest levels of maternal homocysteine were observed in preeclamptic patients with appropriate intrauterine fetal growth. The mean values were $9.004+/-2.820 \mu \mathrm{mol} / \mathrm{L}$ in the IUGR group, $10.815+/-3.785 \mu \mathrm{mol} / \mathrm{L}$ in the group PRE, $9.808+/-2.543 \mu \mathrm{mol} / \mathrm{L}$ in the group PRE-IUGR and $7.639+/-2.728 \mu \mathrm{mol} / \mathrm{L}$ in the control group. These differences were statistically significant in Figure 1.

\section{DISCUSSION}

We revealed higher levels of maternal serum homocysteine in the group of pregnant patients with isolated fetal intrauterine growth restriction in comparison with the control subjects. The concentrations of homocysteine were also higher in both groups of patients with pregnancy complicated by preeclampsia with and without IUGR. The highest levels of homocysteine were observed in preeclamptic women with appropriate-for-gestational-age fetal growth.

Table 1. Analysis of obtained results in studied groups of preeclamptic women with appropriate-for gestational-age fetuses and with intrauterine fetal growth restriction and in pregnant women with isolated IUGR without preeclampsia.

\begin{tabular}{|c|c|c|c|c|c|c|c|}
\hline Data & $\mathrm{CON}$ group & $\begin{array}{l}\text { Statistical } \\
\text { analysis } \\
\text { CON-IUGR }\end{array}$ & IUGR group & $\begin{array}{l}\text { Statistical } \\
\text { analysis } \\
\text { CON-PRE }\end{array}$ & PRE group & $\begin{array}{c}\text { Statistical } \\
\text { analysis } \\
\text { CON-PREIUGR }\end{array}$ & PRE-IUGR group \\
\hline Gravidity & $1.29+/-0.59$ & $p=0.268$ & $1.46+/-0.76$ & $\mathrm{p}=0.060$ & $1.72+/-1.10$ & $\mathrm{p}=0.277$ & $1.46+/-0.95$ \\
\hline Parity & $1.24+/-0.48$ & $\mathrm{p}=0.183$ & $1 . .40+/-0.63$ & $p=0.067$ & $1.56+/-0.87$ & $p=0.364$ & $1.35+/-0.80$ \\
\hline $\begin{array}{l}\text { Maternal } \\
\text { age (years) }\end{array}$ & $29.98+/-3.94$ & $p=0.132$ & $28.54+/-4.40$ & $p=0.143$ & $28.04+/-6.29$ & $p=0.693$ & $29.59+/-3.92$ \\
\hline $\begin{array}{c}\text { Maternal } \\
\text { height }(\mathrm{cm})\end{array}$ & $165.35+/-5.89$ & $\mathrm{p}=0.381$ & $164.00+/-5.30$ & $p=0.438$ & $167.21+/-6.85$ & $p=0.273$ & $163.53+/-4.29$ \\
\hline $\begin{array}{c}\text { Maternal } \\
\text { weight }(\mathrm{kg})\end{array}$ & $76.48+/-11.37$ & $\mathrm{p}=0.020156^{*}$ & $69.17+/-10.80$ & $\mathrm{p}=0.003558^{*}$ & $92.38+/-19.74$ & $\mathrm{p}=0.176$ & $81.74+/-13.24$ \\
\hline $\begin{array}{c}\text { Maternal } \\
\text { BMI }\left(\mathrm{kg} / \mathrm{m}^{2}\right)\end{array}$ & $30.29+/-12.40$ & $\mathrm{p}=0.08047$ & $25.85+/-4.271$ & $\mathrm{p}=0.003511^{*}$ & $33.31+/-5.30$ & $p=0.122418$ & $30.38+/-4.75$ \\
\hline $\begin{array}{l}\text { Age of gestation } \\
\text { (weeks) }\end{array}$ & $38.409+/-1.624$ & $\mathrm{p}=0.000614^{*}$ & $36.931+/-1.990$ & $\mathrm{p}=0.000044^{*}$ & $34.327+/-4.858$ & $\mathrm{p}<0.000001^{*}$ & $33.286+/-3.990$ \\
\hline $\begin{array}{l}\text { Birth weight of } \\
\text { infant }(\mathrm{g})\end{array}$ & $3390.789+/-545.196$ & $\mathrm{p}<0.000001^{*} 2$ & $2016.486+/-420.997$ & $\mathrm{p}=0.000004^{*}$ & $2325.172+/-1176.240$ & $\mathrm{p}<0.000001^{*}$ & $1695.862+/-620.967$ \\
\hline $\begin{array}{l}\text { Homocysteine } \\
\text { levels }(\mu \mathrm{mol} / \mathrm{L})\end{array}$ & $7.639+/-2.728$ & $\mathrm{p}=0.0022^{*}$ & $9.004+/-2.820$ & $\mathrm{p}<0.0001^{*}$ & $10.815+/-3.785$ & $\mathrm{p}<0.0001^{*}$ & $9.808+/-2.543$ \\
\hline
\end{tabular}

Data presented as a mean $+/-\mathrm{SD},{ }^{*}$ statistical significance $(\mathrm{p}<0.05)$. Groups of studied women: CON-healthy normotensive pregnant women $($ controls); IUGR normotensive women with pregnancy complicated by isolated intrauterine growth restriction; PRE-preeclamptic women with appropriate growth fetuses; PRE-IUGR - women with IUGR in the course of preeclampsia. 


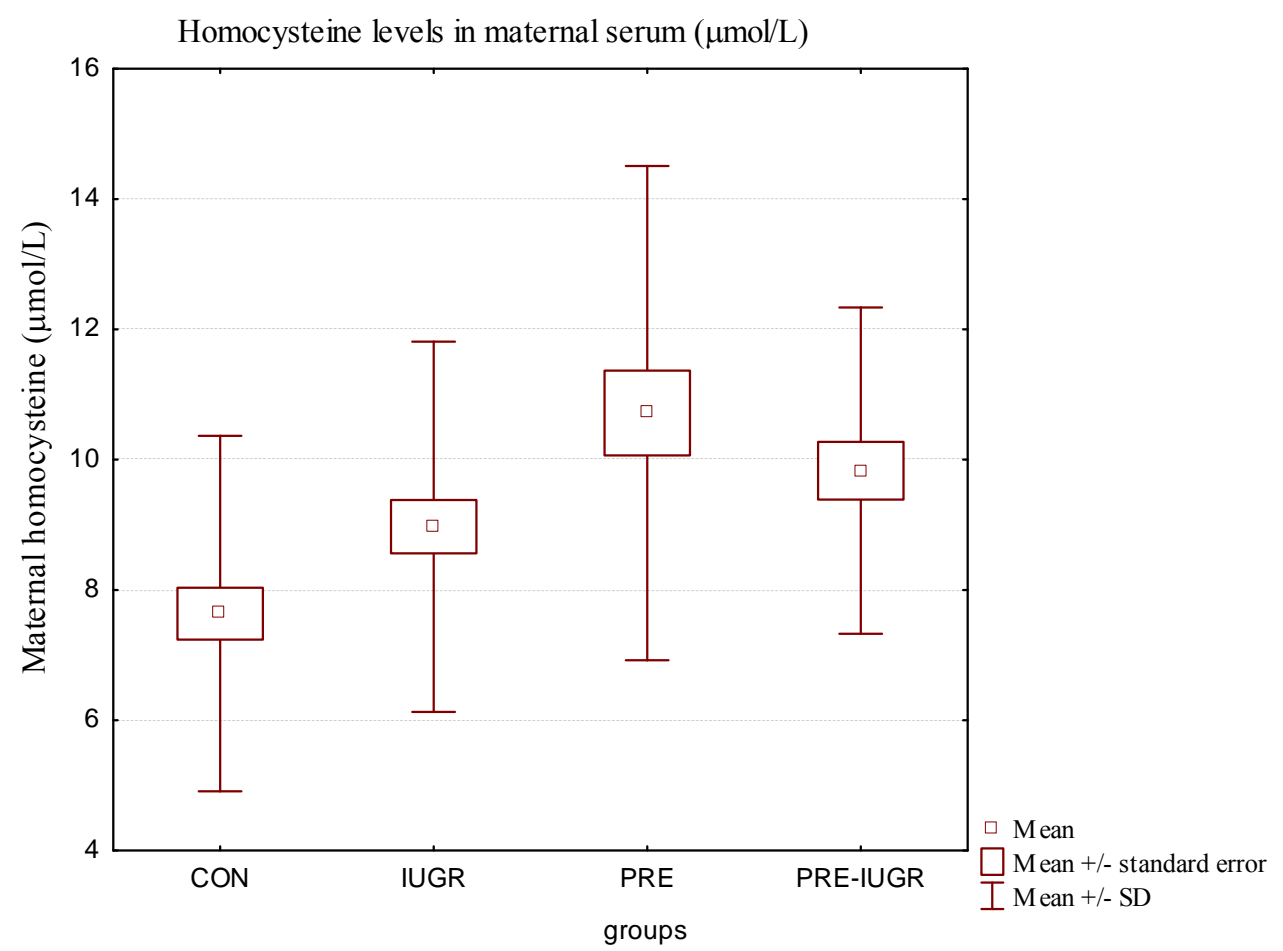

Figure 1. Homocysteine in maternal serum in studied group of women with pregnancies complicated by preeclampsia and/or IUGR.

Also López-Quesada et al. [14] revealed significantly higher homocysteine levels in the preeclamptic women. Similar findings were observed by Wang et al. [15], who demonstrated elevated levels of maternal plasma homocysteine in preeclamptic pregnancies and in pregnancies with suspected fetal compromise and umbilical or placental vascular disease. They concluded that elevated plasma homocysteine plays a role in pathogenesis of the vascular disease in the uteroplacental circulation in placental insufficiency these results are consistent with the hypothesis for the vascular lesion in maternal uteroplacental bed in both preeclampsia and fetal growth restricttion.

Also Mao et al. [16] observed elevated concentrations of homocysteine in patients with severe preclampsia. These authors observed a highly significant positive correlation between the plasma concentrations of homocysteine and asymmetric dimetylarginine (ADMA). They concluded that homocysteine-ADMA-NO pathway may be responsible for etiology in preeclampsia and could be regarded as a marker for the severity of the disease.

According to De Falco et al. [17] hyperhomocysteinemia during pregnancy could be responsible for placental abnormalities, which may be the cause of these very serious pregnancy complications. Also Steegers-Thenissen et al. [18] presented that hyperhomocysteinemia was associated with an approximately 2-fold to 3-fold increased risk of pregnancy-induced hypertension, abrup- tion of placenta and intrauterine growth restriction.

A significant finding in our study is higher levels of maternal serum homocysteine in the group of normotensive pregnant patients with isolated fetal intrauterine growth restriction.

Similar results higher total homocysteine levels in women delivering IUGR infants were presented by Lindblad et al. [19].

But different results were presented by Claire Infante-Rivard et al. [20] who observed in their studies that mothers with small babies had lower homocysteine concentrations than mothers with large babies. Furthermore according to these authors [20] higher maternal homocysteine levels were associated with a statistically significant reduction in risk for IUGR.

Different results in pregnancy complicated by IUGR, but similar results in preeclamptic pregnancies were presented also by Hogg et al. [21]. They analyzed homocysteine levels in maternal plasma in second and third trimester of pregnancies complicated by pregnancy-induced hypertension, preeclampsia and IUGR, respectively. In their studies in these three groups of pregnant women the homocysteine levels in the second trimester of pregnancy did not differ from women with uncomplicated pregnancies in any groups, but at $37^{\text {th }}$ weeks of gestation, the women with pregnancy-induced hypertension or preeclampsia had higher homocysteine concentrations than controls. 
However Hogg et al. [21] did not find such differences in pregnancies with IUGR. They concluded that plasma homocysteine concentrations in the second trimester do not predict the subsequent complications of pregnancy-induced hypertension, preeclampsia and intrauterine growth restriction.

Contrary to our findings also D'Anna et al. [22] didn't find any association between homocysteine levels in pregnancy and IUGR.

Our findings about higher levels of maternal serum homocysteine in pregnancies complicated by IUGR are inconsistent with some earlier reports about the relationship between higher levels of homocysteine in pregnancy with intrauterine fetal growth restriction. This may be due to the fact that we included into a group of women with pregnancy complicated by IUGR only patients with fetal weight estimated below the $5^{\text {th }}$ percentile for gestational age. Furthermore we divided all pregnant patients with fetal growth restriction into two groups, one with IUGR in the course of preeclampsia and the second group with isolated IUGR and normotensive pregnancy, without any maternal or fetal condition responseble for this pregnancy complication. The aim of this was to select the patients with the IUGR due to placental dysfunction.

\section{CONCLUSIONS}

In conclusion, our findings seem to suggest that homocysteine is a mediator of endothelial activation or dysfunction observed in pregnancies complicated by preeclampsia, but also in normotensive pregnancies complicated by isolated IUGR.

It seems that the pathomechanism underlying the development of preeclampsia and/or intrauterine fetal growth restriction is similar, but highest levels of homocysteine observed in preeclamptic pregnancies may suggest that its beginning and intensity may be more enhanced in pregnancy complicated by hypertension and preeclampsia.

Thanks to our better understanding of its physiology and pathophysiology, homocysteine may develop towards a novel target for pharmacological intervention for patients with these pregnancy complications.

\section{REFERENCES}

[1] Mandruzzato, G., Antsaklis, A., Botet, F., Chervenak, F.A., Figueras, F., Grunebaum, A., Puerto, B., Skupski, D. and Stanojevic, M. (2008) Intrauterine restriction (IUGR). Journal of Perinatal Medicine, 36, 277-281. doi:10.1515/JPM.2008.050

[2] Maynard, S.E., Min, J.Y., Merchan, J., Lim, K.H., Li, J., Mondal, S., Libermann, T.A., Morgan, J.P., Selke, F.W., Stillman, I.E., Epstein, F.H., Sukhatme, V.P.M. and Ka- rumanchi, S.A. (2003) Excess placental soluble fms-like tyrosine kinase 1 (sFlt1) may contribute to endothelial dysfunction, hypertension, and proteinuria in preeclampsia. Journal of Clinical Investigation, 111, 600-602.

[3] Maršal, K. (2002) Intrauterine growth restriction. Current Opinion in Obstetrics and Gynecology, 14,127-135. doi:10.1097/00001703-200204000-00005

[4] Robinson, J.S. and Owens, J.A. (1996) Pathophysiology of intrauterine growth failure. In: Gluckman, P.D. and Heymann, M.A., Eds., Pediatrics and Perinatology: The Scientific Basis, Arnold, London, 290-297.

[5] Bretelle, F., Sabatier, F., Blann, A., D’Ercole, Boutiere, B., Mutin, M., Boubli, L., Sampol, J. and Dignat-George, F. (2001) Maternal endothelial soluble cell adhesion molecules with isolated small for gestational age fetuses, comparison with preeclampsia. British Journal of $\mathrm{Ob}$ stetrics Gynaecology, 108, 1277-1282. doi:10.1016/S0306-5456(01)00259-5

[6] Bamberger, A.M., Schulte, H.M., Thuneke, I., Erdmann, I., Bamberger, ChM. and Asa, S.L. (1997) Expression of the apoptosis-inducing fas ligand (FasL) in human first and third trimester placenta and choriocarcinoma cells. Journal of Clinical Endocrinology \& Metabolism, 82, 3173-3175. doi:10.1210/jc.82.9.3173

[7] Hsu, ChD., hariah, H., Basherra, H. and Mor, A.G. (2001) Serum soluble fas levels in preeclampsia. Obstetrics and Gynecology, 97, 530-532. doi:10.1016/S0029-7844(00)01227-8

[8] Roberts, J.M. (1999) Objective evidence of endothelial dysfunction in preeclampsia. American Journal of Kidney Diseases, 33, 992-997. doi:10.1016/S0272-6386(99)70439-7

[9] Granger, J.P., Alexander, B.T., Llinas, M.T., Bennett, W.A. and Khalil, R.A. (2001) Pathophysiology of hypertension during preeclampsia linking placental ischemia with endothelial dysfunction. Hypertension, 38, 718-722.

[10] Kassab, S., Abu-Hijleh, M.F., Al-Shaikh, H.B. and Nagalla, D.S. (2005) Hyperhomocysteinemia in pregnant rats: Effects on arterial pressure, kidneys and fetal growth. European Journal of Obstetrics \& Gynecology and Reproductive, 122, 177-181. doi:10.1016/j.ejogrb.2005.02.008

[11] Aubard, Y., Darodes, N. and Cantaloube, M. (2000) Hyperhomocysteinemia and pregnancy - review of our present understanding and therapeutic implications. European Journal of Obstetrics \& Gynecology and Reproductive, 93, 57-165.

[12] Onalan, R., Onalan, G., Gunenc, Z. and Karabulut, E. (2006) Combining $2^{\text {nd }}$ trimester maternal serum homocysteine levels and uterine artery doppler for prediction of preeclampsia and isolated intrauterine growth restriction. Gynecology and Obstetrics Investigaton, 61, 142148. doi: $10.1159 / 000090432$

[13] Weir, D.G. and Scott, J.M. (1998) Homocysteine as a risk factor for cardiovascular and related disease: Nutritional implications. Nutrition Research Reviews, 11, 311-338. doi:10.1079/NRR19980020

[14] López-Quesada, E., Also-Razo, E. and Vilaseca, M.A. (2003) Hyperhomocysteinemia during pregnancy as a risk factor of preeclampsia. Clinical Medicine, 121, 350-355.

[15] Wang, J., Trudinger, B.J., Duarte, N., Wilcken, D.E. and 
Wang, X.L. (2000) Elevated circulating homocysteine levels in placental vascular disease and associated pre-eclampsia. British Journal of Obstetrics and Gynaecology, 107, 935-938. doi:10.1111/j.1471-0528.2000.tb11095.x

[16] Mao, D., Che, J., Li, K., Han, S., Yue, Q., Zhu, L., Zhang, W. and Li, L. (2009) Association of homocysteine, asymmetric dimethylarginine, and nitric oxide with preeclampsia. Archives of Gynecology and Obstetrics, 1234-1236.

[17] De Falco, M., Pollio, F., Scaramelino, M., Portillo, M. and Lieto, A.D. (2000) Homocysteinemia during pregnancy and placental disease. Clinical \& Experimental Obstetrics \& Gynecology, 27, 188-190.

[18] Steegers-Theunissen, R.P., Van Iersel, C.A., Peer, P.G., Nelen, W.L. and Steegers, E.A. (2004) Hyperhomocysteinemia, pregnancy complications, and the timing of investigation. Obstetrics and Gynecology, 104, 336-343. doi:10.1097/01.AOG.0000129955.47943.2a
[19] Lindblad, B., Zaman, S., Malik, A., Martin, H., Ekström, A.M., Amu, S., Holmgren, A. and Norman, M. (2005) Folate, vitamin B12 and homocysteine levels in South Asian women with growth-retarded fetuses. Acta Obstetricia et Gynecologica Scandinavica, 84, 1055-1061.

[20] Infante-Rivard, C., Rivard, G.E., Gauthier, R. and Théoret, Y. (2003) Unexpected relationship between plasma homocysteine and intrauterine growth restriction. Clinical Chemistry, 49, 1476-1482. doi:10.1373/49.9.1476

[21] Hogg, ChD., Harirah, H., Basherra, H. and Mor, A.G. (2001) Serum soluble fas levels in preeclampsia. Obstetrics and Gynecology, 97, 530-532. doi:10.1016/S0029-7844(00)01227-8

[22] D’Anna, R., Bariera, G., Corrido, F., Lentile, R., Granese, D. and Stella, N.C. (2004) Plasma homocysteine in early and late pregnancies complicated with preeclampsia and isolated intrauterine growth restriction. Acta Obstetricia et Gynecologica Scandinavica, 83, 155-158. 